\title{
publizistischen Aufgabe missionarischer Gemeinschaften
}

\author{
von Franz-Josef Eilers
}

Das Apostolische Schreiben „Evangelii Nuntiandi“, von Papst Paul VI. am 8. Dezember 1975 auch zum zehnjährigen Abschluß des Zweiten Vatikanischen Konzils veröffentlicht, widmet den modernen Kommunikationsmitteln bei den „Wegen der Evangelisierung "1 eigene Aufmerksamkeit. Aber nicht nur in diesem eigenen Abschnitt über die "Benützung der Massenmedien" ist von der Publizistik die Rede. Bereits in dem zweiten der dort insgesamt acht Wege der Evangelisation ${ }^{2}$, bei der Predigt, wird darauf hingewiesen, daß zwar das Wort der Predigt unverzichtbar sei, eine Zivilisation des Bildes uns aber anspornen müsse, „die von dieser Zivilisation hervorgebrachten modernen Mittel für die Vermittlung der Botschaft des Evangeliums einzusetzen “3. Den Stellenwert eines dann noch zusätzlich eingeführten eigenen Abschnitts über die Massenmedien innerhalb des Apostolischen Schreibens für die Evangelisation in der heutigen Zeit mag man ermessen, da der Papst in der Einleitung zum Abschnitt über die Wege der Evangelisierung seine Auswahl damit begründet, daß er nur „auf einige Wege aufmerksam “ machen wolle, „die aus dem einen oder anderen Grunde von entscheidender Bedeutung sind" .

Bei dem Text über die Massenmedien als Mittel der Verkündigung fällt auf, daß der Papst im ersten Abschnitt sich die Stufung des Evangelisationsprozesses in Prä-Evangelisation, Evangelisation und Postevangelisation zu eigen macht, der dann eine Zuordnung der verschiedenen Funktionen der publizistischen Mittel entspricht $t^{5}$ : Es kann „bei der ersten Bekanntmachung mit dem Glauben, bei der katechetischen Unterweisung und bei der weiteren Vertiefung des Glaubens auf diese Mittel nicht verzichtet werden".

Zusammen mit dem Konzilsdekret "Inter Mirifica“ und der Pastoralinstruktion "Communio et Progressio" machen diese weiteren Verlautbarungen die Bedeutung der Kommunikation für die Evangelisation der Kirche von neuem deutlich. Der Papst hält sie für so wichtig, daß er schreibt: „Die Kirche würde vor ihrem Herrn schuldig, wenn sie nicht diese machtvollen Mittel nützte, die der menschliche Verstand immer noch weiter vervollkommnet." Dabei ist er sich durchaus bewußt, daß die Massenmedien eine Seite jenes Prozesses darstellen, der andererseits „immer den Einzelnen innerlich zu treffen" hat, "sich in das Herz eines jeden einsenkt, als wäre er allein in seiner ganzen persönlichen Einmaligkeit ${ }^{\alpha \beta}$.

Bereits aus den Konzilsdokumenten ist deutlich, daß der Einsatz der modernen Kommunikationsmittel nach Meinung der Kirche zunächst in der Verantwortung der Hierarchie und aller ihrer Gliederungen steht ${ }^{7}$. Aber gerade für die Evangelisierung wird man fragen müssen, ob und inwieweit missionarische Gemeinschaften, die z. T. ausschließlich für die Evangelisation gegründet wurden, sich ihrer Verantwortung für die modernen Kommunikationsmittel und ihren Einsatz tatsächlich bewußt sind. Diese Frage stellt sich dabei zunächst weniger an das einzelne Mitglied einer solchen Gemeinschaft und seine persönliche Initiative, sondern vielmehr an die Leitung bzw. an das Gesamtbewußtsein einer solchen Gemeinschaft. Nach den klaren Texten der Kirche ist die Arbeit in und mit den Kommunikationsmitteln nicht eine Sache einzel- 
ner „Fachidioten“, sondern eine Verpflichtung, die im Gesamtbewußtsein einer missionarischen Gemeinschaft ihren anerkannten Platz haben muß. Dies bringt dann natürlich auch entsprechende Folgerungen für die Personalplanung, für die Ausbildung und Arbeitszuweisungen mit sich. Mit zumindest der gleichen Selbstverständlichkeit, wie man die Arbeit der Schulen, der Hospitäler und die Sozialarbeit als missionarische Aufgaben ansieht und aufnimmt, müßte man den Gebrauch und die Einbindung der modernen Kommunikationsmittel in die Evangelisation annehmen.

In einer ersten Phase eines gewissen Enthusiasmus haben einige Generalate missionarischer Gemeinschaften nach dem Konzil in Rom eigene Sekretariate für Kommunikation eingerichtet, ähnlich wie man solche Sekretariate etwa für Erziehung hatte ${ }^{8}$. Bei Sedos, einem Kooperationszusammenschluß von Generalaten missionierender Orden in Rom, gab es in den ersten Jahren, etwa seit 1966, eine eigene Arbeitsgruppe Kommunikation. In der konkreten Arbeit der Kommunikationssekretariate ergab sich fast überall die gleiche Schwierigkeit: Innerhalb ihrer Gemeinschaften wurden sie mehr oder weniger als Pressestelle für den eigenen Orden und als Public RelationsBüro für die Außenwelt angesehen. Tatsächliche Planungsaufgaben für die Aktivitäten der betreffenden Gemeinschaften, zur Bewußtseinsbildung für Kommunikation als besonderer Aufgabe missionarischer Gemeinschaft und integralem Part aller Evangelisationsbemühungen wurden den betreffenden Verantwortlichen nicht gestellt oder bei entsprechenden Vorstellungen kaum ernst genommen. Dies rührte offensichtlich daher, daß die betreffenden Generalleitungen zwar eine Anregung des Konzils äußerlich aufnahmen, innerlich sich aber nicht mit dem identifizierten, was eigentlich gemeint war und jetzt erneut in „Evangelii Nuntiandi“ anklingt. In der Zusammenarbeit der Kommunikationssekretäre mit den betreffenden Ordensleitungen kam z. B. immer wieder die Klage auf, daß man im Grunde von Seiten der Verantwortlichen niche jenes Vertrauen erhalte, das schon für eine solide interne Kommunikationsarbeit notwendig ist. Während im politischen Leben z. B. der Pressechef einer Regierung Kabinettsmitglied ist, um so mit der Kenntnis aller Hintergründe informieren zu können, müssen sich die „Pressechefs" kirchlicher Gemeinschaften oft mit der Sammlung von Nachrichten begnügen, die sie Rundbriefen einzelner Ordensprovinzen oder Mitteilungen einzelner Ordensmitglieder nach eigenem Gutdünken und entsprechend den persönlich entwickelten Beziehungen entnehmen. So ist es verständlich, daß bei etlichen römischen Generalaten die entsprechenden Sekretariate entweder wieder eingegangen oder zur Bedeutungslosigkeit herabgesunken sind. Auch die Kommunikationsarbeitsgruppe bei Sedos besteht nicht mehr, nachdem man sich über längere Zeit fast ausschließlich mit der internen Kommunikation innerhalb der Ordensgemeinschaften beschäftigt hatte. Ausnahme in dieser Entwidklung sind offensichtlich die Jesuiten. Dies ist sicher zu einem großen Teil das persönliche Verdienst des Generaloberen dieser Gemeinschaft, P. Pedro Arrupe. Gerade unter seiner Amtsführung ist innerhalb der Jesuitengemeinschaft ein allgemeines Bewußtsein für die Rolle und Bedeutung der Kommunikationsarbeit in der Kirche gewachsen. Nachdem die 31. Generalkongregation des Ordens (1965/66) ein eigenes Dekret „De mediis Communicationis Socialis" veröffentlicht hatte ${ }^{\mathbf{1 0}}$, nahm der Jesuitengeneral mehrfach zu diesem Thema Stellung ${ }^{11}$. Besonders aufschlußreich ist dabei eine Ansprache von P. Arrupe vor der Versammlung der Prokuratoren des Ordens am 5. Oktober 1970 über das "Apostolat der Gesellschaft in der heutigen Zeit". Dabei nennt Arrupe vier Schwerpunkte für die Arbeit und Verantwortung des Jesuiten heute: 1. die theologische Reflexion, 2. das soziale Apostolat, 3. die Aufgabe der Erziehung und 4. das Apostolat der Massenmedien. Die Bedeutung dieser neuen Mittel, so meinte Arrupe 
damals, könne niemandem entgehen, aber „wir müssen bekennen, daß wir als Söhne der Gesellschaft uns noch nicht wirklich unserer Verantwortung angesichts dieser Realität bewußt sind, die wir ohne Zweifel haben". Im 16. Jahrhundert habe Ignatius, so argumentiert Arrupe u. a., keine Bedenken gehabt, die Kultur und technischen Mittel der damaligen Zeit (z. B. rednerische Fähigkeiten, Theater) zu gebraudhen, was wir auch heute tun müßten ${ }^{12}$. Ein Verzeichnis aus dem Jahre 1975 nennt inzwischen nicht weniger als 560 Jesuiten, die entweder haupt- oder nebenamtlich in irgendeiner Weise in und mit den modernen Kommunikationsmitteln arbeiten ${ }^{13}$.

In diesem Zusammenhang ist es aufschlußreich, daß jetzt mit Berufung auf das Apostolische Schreiben „Evangelii Nuntiandi“ die Millhill Missionare (St. Joseph's Missionary Society) bei ihrem Generalkapitel 1976 in Millhill bei London, dem Sitz der Generalleitung, für die zukünftige Arbeit ihrer Gemeinschaft drei Schwerpunkte festgelegt haben: 1. das Apostolat für die Armen in den Städten, 2. das Apostolat des Gebetes und 3. den Gebrauch der modernen Kommunikationsmittel. Dabei soll es vor allem um den Einsatz geschulten Personals als Dienstleistung für die jungen Kirchen in der Dritten Welt gehen ${ }^{14}$. Hier fällt auf, daß mindestens zwei dieser drei Schwerpunkte den von $P$. Arrupe für die Jesuiten gesetzten Schwerpunkten sehr ähnlich sind, nämlich die Armen in den Städten (Soziales Apostolat) und die Kommunikationsmittel.

Für die Dominikaner ist die Behandlung der Kommunikationsarbeit für das nächste Ordenskapitel Ende 1977 in Manila als Thema der Zusammenkunft vorgesehen. Nachdem bereits früher mit einem Publizistiksekretariat des Ordens in Paris begonnen worden war, das eine Auflistung solcher Mitglieder veröffentlichte, die in diesem Bereich tätig sind, hat der Generalmagister eine kleinere Gruppe solcher Ordensmitglieder im Frühjahr 1977 nach Rom berufen, um die Besprechungen zum Thema in Manila vorzubereiten ${ }^{15}$.

Neuerdings hat die römische Union der Generalsuperioren ihre Mitglieder bzw. die stellvertretenden Generaloberen zu einem Studientag über „Erziehung zur Sozialen Kommunikation" eingeladen ${ }^{16}$. Ob diese Entwicklungen als Anzeichen einer neuen Bewußtseinsbildung innerhalb missionarischer Gemeinschaften zu ihrer publizistischen Verpflichtung in dieser Zeit anzusehen sind, muß die Zukunft erweisen. Das Bemühen um die modernen Kommunikationsmittel ist jedenfalls weit mehr als eine Frage der Kommunikation innerhalb der betreffenden Gemeinschaften oder ihrer Präsentation vor der Außenwelt. Es geht heute um eine tiefere Einsicht in den Kommunikationsprozeß als solchen in all seinen Verästelungen und deren Bedeutung für die Kommunikation der Menschen untereinander und mit Gott. Dies erfordert eine ernste fachliche Ausbildung und Auseinandersetzung. Ein erster Schritt dazu wäre konkret etwa eine Auflistung aller Mitglieder einer missionarischen Gemeinschaft, die bereits in der einen oder anderen Weise in diesem Bereich tätig sind. Dies sollte dann möglichst so geschehen, daß auch deutlich wird, a) welche Ausbildung der Betreffende hat, b) worin seine Arbeit besteht und c) welche Verantwortung er innerhalb dieser Arbeit trägt ${ }^{17}$. Ferner müßte im Rahmen der allgemeinen Ausbildung eine entsprechende Einführung und Weiterbildung im Bereich der Kommunikationsmittel gegeben werden. Diese Forderung steht zwar schon in den kirchlichen Dokumenten und inzwischen auch in einigen Ordensregeln ${ }^{18}$, wird aber praktisch kaum durchgeführt. Auch könnte man dabei entsprechende Talente entdecken, was wiederum zu der weiteren Forderung nach einer entsprechenden Personalplanung führen würde. Gerade weil der Nachwuchs schwächer wird, sind eine in die Zukunft weisende Personalplanung und gezielte 
fundierte Ausbildung notwendig. Im übrigen können missionarische Gemeinschaften nur dann wirklich im Bereich der Kommunikationsarbeit etwas leisten und mitreden, wenn sie ihre Mitglieder qualitativ so gut ausbilden, daß sie auch im nichtkirchlichen Raum fachlich anerkannt werden. Schließlich sollten missionarische Gemeinschaften ihre Aufgabe in der Schulung von Fachkräften sehen. So wie Lehrerbildungsanstalten, Akademien und Universitäten eine Selbstverständlichkeit sind, sollten Kommunikationsausbildungszentren für Christen und Nichtchristen eine besondere Aufgabe einer missionarischen Gemeinschaft sein. Wer heute die Kommunikatoren ausbildet, bestimmt entscheidend das Bild der Gesellschaft von morgen. Auch regelmäßige Treffen und gegenseitige Information aller Mitglieder, die innerhalb einer Gemeinschaft in diesem Bereich tätig sind - sei es auf nationaler, kontinentaler oder Weltebene - würden das Bewußtsein für die missionarische Aufgabe der Kommunikation stärken ${ }^{19}$.

\section{Anmerkungen:}

1. Teil IV des Schreibens.

2. „Evangelii Nuntiandi“ Nr. 40-48: 1. Das Zeugnis des Lebens, 2. Eine lebendige Predigt, 3. Wortliturgie, 4. Katechese, 5. Massenmedien, 6. Persönlicher Kontakt, 7. Die Rolle der Sakramente, 8. Volksfrömmigkeit.

3. „Evangelii Nuntiandi" Nr. 42.

4. Ebd. Nr. 40.

5. Vgl. hierzu u. a. Franz-Josef Eilers: Zur Bedeutung der Publizistik in der Missionsarbeit heute. In: „Neue Zeitschrift für Missionswissenschaft“, Beckenried 28:1972, 241-252; Ders.: Kommunikation und Evangelisation: ein Diskussionsbeitrag. In: CS 8:1975, $201-212$.

6. „Evangelii Nuntiandi“ Nr. 45. Hier wird deutlich, daß es sich bei den Kommunikationsmitteln nicht um einen oberflächlichen Massenprozeß handeln darf: Immer wieder ist die Rückkoppelung in Verantwortung und Entscheidung des Einzelnen notwendig, die auch in der modernen Kommunikationsgesellschaft den persönlichen Kontakt unersetzlich macht. Vgl. dazu auch Nr. 46 von „Evangelii Nuntiandi“.

7. Vgl. z. B. "Inter Mirifica" Nr. 13; 19-22.

8. Z. B. so bei den Oblaten von der Unbefleckten Empfängnis OMI, bei den Karmeliten, den Steyler Missionaren und den Jesuiten.

9. Eine Initiative der Päpstlichen Kommission für die Sozialen Kommunikationsmittel mir der Einladung von Kommunikationssekretären der Generalate zu einer gemeinsamen Konferenz blieb vor einigen Jahren wohl ein einmaliges Ereignis. Von Msgr. Deskur, dem Präsidenten der Päpstlichen Kommission für die Sozialen Kommunikationsmittel, wird berichtet, er habe gesagt, $70 \%$ aller in der Kirche im Kommunikationsapostolat tätigen Menschen seien Ordensleute (OCIC Newsletter 1977/2, S. 9). Dies kann durchaus zutreffen, wenn man die einzelnen Ordensleute nimmt und Gemeinschaften, die ganz für dieses Apostolat gegründet wurden, wie etwa die verschiedenen Gruppierungen der Paulisten (vgl. Karl Vospernik: Eine Ordensfamilie für publizistisches Apostolat: die Pauliner. In: CS 5:1972, 19-26). Tatsächlich sind etwa in Asien und Afrika die meisten kirchlichen Kommunikationsverantwortlichen Jesuiten. Hier geht es aber um eine Gesamteinstellung missionarischer Gemeinschaften, die mehr sein sollte als eine Sammlung von Einzelinitiativen.

10. Der volle Text des Dekretes lautet:

Decreto 35. "De mediis communicationis socialis"

"Praesentia "et influxus novorum mediorum communicationis socialis (radiophoniae, cinematographiae, televisionis) in mundo hodierno intensitate et extensione in dies augentur. 
Ex una parte constituunt nostro tempore expressionis instrumenta maximi momenti ideoque nobis praebent aptissima apostolatus subsidia ad plura ministeria Societatis.

Ex alia vero parte talium mediorum diffusio in universa familia humana ita mentes et animos cuiusque aetatis et condicionis afficit, ut vere influxum sane universalem exerceant et proinde determinent quid cogitent, immo quid agant hodierni homines.

2. Unde sequitur quod haec media non amplius considerari oportet ut primario directa ad animi relaxationem, sed potius ut media expressionis et communicationis socialis in praesenti hominum rerumque condicione, quae quodammodo appellari potest „cultura imaginis".

3. Congregatio Generalis iuxta decretum Concilii Vaticani II de mediis communicationis socialis ordinationes priores recognoscens, haec de apostolatu novorum illorum communicationis mediorum commendare vult:

$1^{\circ}$ Adhibeantur haec media communicationis socialis utpote instrumenta valde efficacia in pluribus e nostris ministeriis, et speciatim in praedicatione verbi Dei et in institutione iuventutis.

$2^{\circ}$ Etam ratio habeatur de specifica opportunitate ab illis mediis provisa, sive quaestiones tractentur, sive personae excolantur, quae non facile aliis mediis et formis apostolatus attingi valeant.

$3^{\circ}$ Provideatur ut in curriculo formationis nostrae tradatur disciplina de mediis communicationis socialis, unicuique periodo formationis accommodata.

$4^{\circ}$ Seligantur tempestive a Provincialibus aliqui qui spiritu religioso et aliis dotibus apti sint ut, vario gradu specializationis periti et titulis academicis ornati, competentes fiant in hoc apostolatu exercendo et aliis in ipso dirigendis. Ubi autem opportunum fuerit, aliquod centrum instituatur quod iuvent ad hanc specializationem acquirendam.

$5^{\circ}$ Quae ab A. R. P. Janssens de secretariatibus (mundiali, regionali et provinciali) statuta sunt, fideliter exsecutioni mandentur, utpote medium necessarium et efficax ad hoc genus apostolatus urgendum et promovendum." (A. R. Vol. XIV, 1961 -1966, p. 967)

11. So z. B. in einer Rede zur Eröffnung einer Versammlung von SJ-Kommunikationsspezialisten in Rom vom 7. bis 13. März 1966 und zur Eröffnung eines Jesuiteninstituts für Massenmedien in Detroit am 3.8.1966. Beide Dokumente in: Ramon Aguiló: Formation of Jesuits in Social Communications. Texts in their original languages. Vervielfältigt, Rom 1969, 14-19. - Vgl. ferner die Intervention von Arrupe bei der Römischen Synode 1975 über Offentlidhe Meinung und Evangelisation. In: CS $8: 1975$, 165-169.

12. Der Wortlaut des entsprechenden Abschnittes lautet:

"- $4^{\circ}-$ Quartum est genus ministerii, quod simul via ideas diffundendi et hodierni mundi educationem promovendi dicendum est, quodque maximum nunc influxum exercet in nostris coetaneis et optimum apparet ad ipsum evangelium efficaciori modo praedicandum. Loquor de illis sic dictis Mass Media, quae essentiales hodie partes exercent in toto humano commercio, et a quibus dependet informatio, delectatio, modus cogitandi et agendi non exiguae partis totius generis humani. Neminem latet momentum huius novae humanae inventionis, sed tamen confiteri debemus nondum nos, ut societatis filios, vere conscios factos fuisse nostrae indubiae responsabilitatis coram evidenti hac realitate. Medium enim prae manibus habemus, quo innumeram hominum multitudinem attingere poterimus, si ea sciverimus patienter uti.

Saeculo decimosexto Societas nostra non dubitavit illius aetatis culturam et technica media paenitus accipere et adhibere: Patres nostri et Scholae Societatis coluerunt humaniores litteras, oratoriam artem, scaenicas repraesentationes, omnis generis technicam illius temporis evolutionem, nihil recusantes, sed e contra omnia adhibentes in proposito mundum Christo lucrifaciendi. Quod Ignatius, Xaverius et tot alii e primis nostri Patribus fecerunt, idem et nos facere debemus.

Societas nostra serio ac diligenter hoc punctum perpendere debet, ne veteribus habitudinibus gravata mobilitatem amittat charismatis primigenii.

Vestrae considerationi, cari Patres, et per vos considerationi vestrarum Provinciarum committere velim hanc meam sollicitudinem, qua iudicaverim nos multo plure efficere posse in servitium animarum si his modernis apostolatus intrumentis recte uti didicerimus, si illa Mass. Media et quotquot in eis laborant partem hodierni nostri apostolatus consideraverimus, si denique collaborationem nostram obtulerimus praeparandis, adiuvandis, dirigendis innumeris illis hominibus, qui istiusmodi mediis communicationis dant operam.

Nec vos fugit quam haec media utilia nobis esse poterunt pro ipsa Nostrorum efforma- 
tione et institutione, prout iam luculenter probant experimenta facta in nonnullis provinciis.

Scio rem difficultatibus non carere nec fieri posse sine diligenti studio, sed experientia iam constat quanta hic labor mercede compensetur, si debito modo fiat et apta media adhibeantur ad finem quem intendimus persequendum ...."

13. Ramon Aguiló: Jesuits in Mass Media, Rom 1975. Vgl. Rezension CS 9:1976, $184 \mathrm{f}$.

14. Documents of the General Chapter 1976, St. Joseph's Missionary Society of Mill Hill. Vervielfältigt, London 1976, 19-22.

15. Catalogus Fratrum Ordinis Praedicatorum et Sororum Familiae Dominicanae in Mediis Communicationis Socialis Laborantium, Paris o. J. (1976); vgl. Praedicare con i mass media, Interview des Vatikansenders mit P. Reinfried Buetho, „Radiogiornale “, Vatikansender vom 3. Mai 1977.

16. Je nach Sprachgruppe der Generalate vorgesehen für den 21. und 22. April 1977 in Rom. Vgl. „Radiogiornale“, Vatikansender v. 15. 4. 1977.

17. Vgl. etwa die Aufstellung der Jesuiten, s. Anmerkung 13.

18. Z. B. bei den Steylern und Oblaten (OMI).

19. Dies geschieht bereits bei den Jesuiten, wo unter der Bezeichnung ,Jescom' (= Jesuits in Communication) die betreffenden Mitglieder national, kontinental und interkontinental (z. B. Jescom-India, Jescom-Asia, Jescom-International) zusammenarbeiten und auch z. T. gemeinsam Projekte durchführen.

\section{SUM M A R Y}

In "Evangelii Nuntiandi" of the 8th December, 1976, under the section on methods of evangelisation there is reference to the use of modern communications media. In a further subsection on preaching the Pope speaks of the use of modern media in "transmitting the message". Together with the Council document "Inter Mirifica" and the Pastoral Instruction "Communio et Progressio", we have here clearly set out the importance of communications for evangelisation. A certain enthusiasm in some Roman Generalates after the Council faded rather quickly as superiors were apparently not prepared to take concrete, practical action. There now seems to be hope for new initiatives. These should, for example, start with a listing of all those members of communities already working in the field of communications. There should be systematic training leading to qualifications, and planning for the personnel concerned. Training facilities for future communicators in the secular field also should be seen as an eminent duty appertaining to communities dedicated to evangelisation.

\section{$R E S U M E$}

Dans l'écrit apostolique »Evangelii Nuntiandi« du 8 décembre 1975 parmi les »voies de l'Evangélisation « se trouve un paragraphe propre dédié aux moyens de communication modernes et dans le sermon il est également question des moyens modernes pour la "propagation de la Bonne Nouvelle«. Avec le décrêt conciliaire "Inter Mirifica«, de pair avec l'instruction pastorale "Communio et progressio « apparait clairement ici la signification de la communication pour le travail d'évangélisation de l'Eglise. Ceci devrait avoir des conséquences particulières pour ces communautés ecclésiastiques qui ont été fondées spécialement pour cette tâche. Après un certain enthousiasme chez quelques généralats de telles communautés après le Concile qui recommençait bientôt à faiblir parce qu'on ne voulait visiblement pas tirer des conséquences concrêtes pratiques, on doit espérer maintenant que de nouvelles initiatives seront prises. Ceci pourrait commencer par un démembrement de tous 
ceux qui sont déjà actifs dans ce domaine; à cela devraient s'ajouter une formation systématique qualifiée et une planification correspondante du personnel. De même, il devrait être créé des possibilités de formation pour les futurs communicateurs, dans le domaine non religieux. Ici repose une tâche pour les communautés missionnaires.

\title{
RESUMEN
}

El escrito apostólico "Evangelii Nuntiandi« del 8 de Diciembre de 1975 incluye entre los "caminos de la evangelización « un apartado propio para los medios de difusión. También al tratar de la predicación se habla de los medios modernos para la »transmisión del mensaje«. Junto con el decreto conciliar "Inter Mirifica y la instrucción pastoral »Communio et Progressio se pone aquí de manifiesto la importancia de la comunicación en el trabajo evangelizador de la Iglesia. Ello debe tener consecuencias especiales para las sociedades eclesiásticas creadas con ese fin. Después de cierto entusiasmo post-conciliar en algunas casas generalicias de tales sociedades, que se esfumó pronto tal vez porque no se querían sacar las consecuencias prácticas concretas, es de esperar que se produzcan ahora nuevas iniciativas. Podría empezarse por la puesta al día de quienes trabajan en ese sector; por otra parte debería llegarse a una formación sistemática, cualificada y a la correspondiente planificación del personal. Deberían crearse posibilidades de formación también para futuros profesionales de la comunicación en sectores no eclesiales. Éste es cometido de las sociedades misioneras.

\section{Zum Einfluß von Gewalt im Fernsehen auf die Gewissensbildung Jugendlicher: pastorale Überlegungen}

\author{
von Paul Nikolajczyk
}

Das Zweite Vatikanische Konzil richtet in seinem am 4. Dezember 1963 verkündeten Dekret über die sozialen Kommunikationsmittel "Inter Mirifica“ seine besondere Aufmerksamkeit vor allem auf Presse, Film, Rundfunk und Fernsehen. Als Voraussetzung zur Benutzung der sozialen Kommunikationsmittel nennt das Konzil sowohl die Kenntnis der Grundsätze sittlicher Wertordnung als auch die Bereitschaft, sie hier zu verwirklichen. Warnend weist das Konzil gleichzeitig darauf hin, daß die Eindruckskraft der sozialen Kommunikationsmittel so groß sein kann, daß es den Menschen, insbesondere den unerfahrenen, schwer fällt, sich ihrer bewußt zu werden, sie geistig zu verarbeiten und gegebenenfalls sich ihrer $\mathrm{zu}$ erwehren. Jugendliche und Kinder haben das Recht darauf, angeleitet zu werden, die sittlichen Werte mit richtigem Gewissen zu schätzen und in personaler Bindung zu erfassen ${ }^{1}$. Diesen Anliegen des Konzils möchte dieser Aufsatz am Beispiel „Gewaltszenen im Fernsehen“ (kurz:

Paul Nikolajczyk, Dipl.-Theol., studierte nach einer Tätigkeit als Industriekaufmann Theologie in Paderborn und Münster; er ist Vikar in Castrop-Rauxel. 
Mediengewalt) auf mögliche Einwirkungen bei der Gewissensbildung der Jugendlichen entsprechen, zumal unterschiedliche Definitionen der religiösen und sozialen Reife in Kirche und Staat (Religionsmündigkeit, Berufs-, Ehe-, Straf-, Wahl- und Wehrreife) dem Jugendlichen die religiöse und soziale Orientierung erschweren und ihn mit Rollenkonflikten und Orientierungsschwierigkeiten konfrontieren. Die katholische Kirche, die gemäß den Anfangsworten der am 15. Mai 1961 von Papst Johannes XXIII. veröffentlichten Sozialenzyklika „Mater et Magistra“ den Anspruch erhebt, „Mutter und Lehrerin" zu sein, darf den Jugendlichen beim Erkennen seines Reifungsstandes, bei der adäquaten Aufarbeitung seiner Reifungsrückstände und bei der Hilfe zur Erreichung der natürlichen und übernatürlichen Vollendung nicht allein lassen. Das Fernsehen hat hier zusätzliche Chancen und Gefahren gebracht.

Als 1843 der schottische Psychologe und Logiker Alexander Bain (1818-1903) auf die Notwendigkeit verwies, elektrisch zu übertragende Bilder punkt- und zeilenweise abzutasten, war es noch ein weiter Weg, bis es dem deutschen Ingenieur P. G. Nipkow 1884 gelang, mit spiralisch angeordneten quadratischen Löchern die Aufnahme sich bewegender Bilder zu ermöglichen. 1906 wurde dieses System von Max Dieckmann durch Verwendung der Braunschen Kathodenstrahlung vervollständigt. Die ersten regelmäßigen Fernsehsendungen wurden 1928 mit einem von der Firma General Electric entwickelten System über die Station WGY in Schenectady ausgestrahlt. Für Deutschland wurde am 24. Dezember 1930 in Berlin der Fachwelt das erste vollelektronische Fernsehbild vorgeführt; der regelmäßige Fernsehprogrammdienst begann am 22. März 1935 in Berlin. Nach den Wirren des Krieges wurde am 22. September 1950 der erste Fernsehsender für Deutschland in Hamburg in Betrieb genommen. Rechtliche Grundlage bilden Gesetze und Staatsverträge, die das Recht auf Selbstverwaltung garantieren. Die weite Verbreitung des Fernsehens ${ }^{2}$ hat das menschliche Leben nachhaltig beeinflußt: Besuchs-, Essens- und Zubettgehensgewohnheiten, unkontrollierte Aufnahme von Getränken, Speisen und Rauchwaren, Gefahr der verkürzten Nachtruhe wegen der Unfähigkeit vieler Zuschauer, spannende Filme abzuschalten; positiv aber u. a. auch, daß das Fernsehen die Mehrheit der Haushalte zur Teilnahme an den Problemen und Lösungsmöglichkeiten religiöser, gesellschaftlicher und politischer Fragen im In- und Ausland ermutigt; außerdem wertvolle Kulturund Unterhaltungsbeiträge sowie Chancen zur Bildung durch das Telekolleg. $\mathrm{Zu}$ den beliebtesten Sendungen zählen die Kriminalfilme ${ }^{3}$. Aufschlußreich ist auch der durchschnittliche Zeitaufwand für das Fernsehen in der Bundesrepublik Deutschland nach Stunden und Minuten (1970).

\begin{tabular}{lcccccc}
\hline Altersgruppen & \multicolumn{2}{c}{ Montag bis } & Freitag & \multicolumn{2}{c}{ Samstags } & \multicolumn{2}{c}{ Sonntags } \\
& Sommer & Winter & Sommer & Winter & Sommer & Winter \\
\hline 14-19 Jahre & 1.41 & 1.32 & & & & \\
20_29 Jahre & 1.31 & 1.32 & 1.56 & 2.14 & 2.34 & 2.26 \\
30-39 Jahre & 1.20 & 1.29 & 2.03 & 2.32 & 2.02 & 2.53 \\
40_49 Jahre & 1.23 & 1.37 & & & & \\
50_59 Jahre & 1.36 & 1.32 & 2.26 & 2.30 & 2.02 & 2.46 \\
60 Jahre und mehr & 1.50 & 1.49 & & & & \\
\hline Gesamt & 1.32 & 1.36 & 2.08 & 2.26 & 2.17 & 2.43
\end{tabular}


1974 hatte ein bundesdeutscher Durchschnittsbürger pro Werktag 2:11 Stunden fürs Fernsehen übrig, sofern er in einem Haushalt mit TV-Gerät (95 von 100) lebte 4 .

Angesichts dieser statistischen Ubersicht werden die Anschauungen des Zweiten Vatikanischen Konzils verständlicher: die katholische Kirche versucht ihren Beitrag zu leisten, um aus dem Geist der Heiligen Schrift in der lebendigen Verkündigung dem Jugendlichen Hilfen zur Gewissensbildung gegenüber der Mediengewalt zu geben.

Ein erster Schritt hierzu ist die Verdeutlichung des christlichen Begriffes der „Gewalt“. Nach dem Zeugnis des Neuen Testaments bekennt Jesus, daß ihm alle Gewalt (exousía) sowohl im Himmel als auch auf Erden gegeben ist (Mt 28,18). Der Mensch, der sich durch Glauben und Taufe Christ nennen darf, weiß, daß er im Himmel

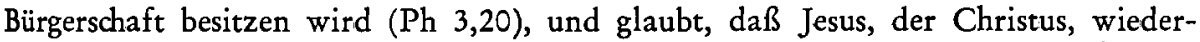
kommen wird, um das Heil zu vollenden. Daher lehnt der Christ das Aufgehen im Besitz irdischer Güter $a b$ und relativiert gleichzeitig das Streben nach Macht und Gewalt sowie das Anwenden und Abhängigsein von ihnen. Ermutigt wird er hier durch das Beispiel seines Herrn, um dessen tägliche Nachfolge er sich bemüht (Joh. 12,26): Jesus Christus hat, um die Welt zu „erlösen“, keine Gewalt angewendet und somit nicht andere, sondern sich selbst "ge-opfert". Vertreter von Ideologien hingegen, die das „Heil“ ausschließlich durch das Handeln des Menschen, ohne Gott und somit bewußt und militant a-theistisch, für erreichbar und innerhalb dieser Welt für realisierbar halten, werden kaum Bedenken haben, unter gewissen Umständen zur "gewaltsamen "Veränderung „ungerechter" Strukturen aufzurufen und mitzuarbeiten. Für einen Christen hingegen eröffnet sich in der Einübung der Tugend der Geduld eine andere Sicht: Hier wird vorausgesetzt, daß das Gleichnis von der „selbstwachsenden Saat" (Mk. 4,26 f.) auch heute aktuell ist. Stärker als bisher könnte die Kirche in ihrer Verkündigung darauf aufmerksam machen, daß zu den Kerngeheimnissen des Glaubens die Tatsache zählt, daß Gott da ist und jeden einzelnen Menschen bejaht, so daß dieser sich in allen Lebenssituationen als unendlich geliebt wissen darf. Insbesondere Kriminalfilme neigen jedoch zu übereilten Lösungen, die das Einüben in Geduld und somit in ein verantwortbares Gottvertrauen erschweren und sogar bisweilen verhindern.

Da das Gottesbild das Bild vom Menschen beeinflußt, andererseits aber auch ein Menschenbild ohne Gott möglich ist, könnte die Kirche hier neue Wege gehen, um auf dem scheinbar a-theistischen Terrain „Mediengewalt und jugendliche Gewissensbildung" die frohmachende Botschaft besser verkündigen zu können.

Zur Verdeutlichung zeitgemäßer Theorien über den Ursprung von Gewalt, mit denen sich die Kirche auseinanderzusetzen hat, seien drei genannt:

\section{Gewalt als Folge eines angeborenen Aggressionstriebs}

Hier wird auf das „Dampfkessel-Modell“ verwiesen, welches die unbewußte Seele als brodelndes Reservoir chaotischer Triebenergien deutet. Diese gestauten Triebenergien müsse man auf irgendeine Art „ablassen“, damit keine Katastrophe entstehe. Diese Theorie kann in Konflikt mit dem christlichen Liebesgebot stehen, wie es das Beispiel Alexander Mitscherlichs zeigt: „In Wahrheit ist aber die Forderung nach uneingeschränkter Nächstenliebe undurchführbar. "5 Zusammen mit dem jetzigen 
Bischof von Aachen, Klaus Hemmerle, versuchte W. Hagemann hierzu aus katholischem Verständnis in direkter Anlehnung an Mitscherlich eine Antwort: „Der Mensch ist hier überfordert und steht vor einer Grenze... Aber diese Grenze ist in Christus aufgebrochen worden... Von ihm her ist Menschlichkeit möglich. "6 Ergänzend sei vermerkt, daß die geläufige UUbersetzung „Auge um Auge und Zahn um Zahn" insofern Verwirrung erzeugen kann, als ein gewalttätiger Gott des Alten Bundes im Gegensatz zum „lieben“ Gott des Neuen Bundes gesehen wird. Martin Buber (18781965) über-setzt „Augersatz“ für Auge und „Zahnersatz“ für Zahn"a. Wenn auch der Begriff Zahnersatz als feststehender medizinischer Begriff an dieser Bibelstelle überholbar sein dürfte, so kommt doch bei Buber der Gerechtigkeitsgedanke beim Vergeltungsdrang besser heraus, und es wird verhindert, daß Jahwe als „DraufgängerGott" gesehen werden könnte.

\section{Gewalt als Folge von Frustration}

Unter Frustration („Lust-Verlust“) ist hier im weitesten Sinne Enttäuschung durch Ausbleiben eines ersehnten Erfolges gemeint. Philipp Lersch (1898-1972) zeigt die Beziehung von Hoffnung und Enttäuschung auf: „Nur wer hofft, kann enttäuscht werden. ${ }^{\text {" } 7}$ Uber das Wesen der Frustration gibt es unterschiedliche Auffassungen: „Zwei verschiedene Einstellungen zur Untersuchung der Frustration werden deutlich. Erstens gibt es eine breitangelegte Reihe von Untersuchungen, die Frustration mit Aggression, Regression und Fixierung verknüpfen. An zweiter Stelle ist eine große Anzahl von Laborarbeiten zu nennen, die ihr Augenmerk auf die Entstehung von Trieb- und Hinweisreizen durch frustrierende Nichtbelohnung richten. Die zuletzt genannten Untersuchungen fanden später als die ersteren statt, und es hätte erwartet werden können, daß ein fruchtbarer Dialog $z$ wischen den Standpunkten auf eine Wiederbelebung des Interesses an den Frustrations-, Aggressions-, Regressions- und Fixierungs-Hypothesen hinausgelaufen wäre. Dieser Dialog hat zum Schaden der Sache noch nicht eingesetzt. ${ }^{\text {" }}$ Ungeachtet dieses wissenschaftlichen Streites könnte die Kirche ihre speziellen Hilfen u. a. durch den Hinweis auf Heiligenleben anbieten, zumal sie in der Konzilskonstitution über die Liturgie "Sacrosanctum Concilium“ die Heiligenverehrung etwa damit begründet, daß sie den Gläubigen ein Beispiel vor Augen stellt, das alle durch Christus zum Vater zieht ${ }^{9}$. Hier könnte auch "narrative Theologie“ (J. B. Metz) geschehen. Insgesamt müßte es der Kirche, keineswegs nur in ihrem "Amt", mehr denn je gelingen, „frustrierende Situationen" durch die Hoffnungsstruktur des christlichen Glaubens zu bewältigen.

\section{Gewalt ist erlernbar und verlernbar}

Zur Verdeutlichung dieser These könnte ein mögliches Bonmot einer Werbe-Agentur dienen: „Ich will, daß Du willst, was ich will, ohne daß $D u$ merkst, daß ich es will!" Insgesamt kann davon ausgegangen werden, daß beispielsweise die Werbung stark auf die Macht der "geheimen Verführer" baut. Wenn der Mensch sich Vorbilder sucht, könnte hier die Kirche aus ihrer reichen Geschichte das Leben von Heiligen, gerade in ihrer Zeitbedingtheit, den suchenden Menschen und Jugendlichen näherbringen. Falsch ist es, wenn die Kirche nur schlechte Filme verurteilt, ohne Alternativen anzubieten. 
Nach diesen Darstellungen über den Ursprung von Gewalt seien die vier bekannten Theorien über Wirkungen der „Mediengewalt" dargestellt und erläutert:

\section{Katharsis-These}

Diese These setzt die Richtigkeit des „Dampfkessel-Modells“ voraus. Ungeachtet der Feststellung von L. Berkowitz, sie sei in ihrer traditionellen Form widerlegt ${ }^{10}$, muß darauf aufmerksam gemacht werden, daß diese These auf keinen Fall hilft, die Ursachen der Gewalt zu erkennen und adäquat zu beseitigen, oder Hilfen gibt, trotz erkannter, aber nicht behebbarer Ursachen nicht zu verzweifeln, sondern zu leben. Eine Antwort der Kirche zur Katharsis-These könnte u. a. darin bestehen, Menschen, die, aus welchen Gründen auch immer, verzweifelt sind, zu helfen, den Teufelskreis ihrer Verzweiflung zu durchbrechen. Dies könnte etwa in einem Angebot zu einem Seelsorge- oder Beichtgespräch führen, wo der einzelne den Mut haben darf, das Kreisen um sich selbst einzugestehen, wo er dann keine Rollen zu spielen und keine Masken zu tragen braucht, weil er sonst ängstlich meinen müßte, er sei nicht mehr „in “. In einem solchen Gespräch dürfte dieser Mensch einmal er selbst sein. Er könnte über sich sprechen, weil er weiß: Hier hört ein verstehender Mensch mir zu, und hier werde ich so angenommen, wie ich wirklich bin. Es gibt eine eigenartige Beobachtung: Sobald ein Mensch etwas falsch gemacht hat, neigt er dazu, die "Schuld" auf andere zu schieben, und sobald etwas gelungen ist, ist der Mensch nicht selten stolz auf sich. Die Kirche könnte hier das alttestamentliche Wahrhaftigkeitsgebot des Dekalogs neu entdecken helfen, wo die größte Lüge darin besteht, daß jemand sich selbst belügt, ohne es zu merken und so ständig auf der Flucht vor sich selbst ist. Theologisch ist hier der Ort, das end-gültige Heilshandeln Gottes an uns in Jesus Christus zu verkündigen: Weil Gott uns bejaht, brauchen wir selbst uns nie auszuweichen. Hätte die Katharsis-These recht, daß durch Mediengewalt ein Abbau aggressiver Emotionen und Verhaltenstendenzen durch Identifikation mit der dargestellten Gewalt erreicht werden könnte, so würden dem Menschen viele Möglichkeiten genommen, sein wahres Leben zu entdecken und dauerhafte Lösungen zur Konfliktbewältigung zu erstreben.

\section{Inbibitions-These}

Nach dieser These wirkt Mediengewalt hemmend und abschreckend auf die Äußerung aggressiver Emotionen und Verhaltenstendenzen. „Obwohl die ,bösen“ Aggressoren ofter als die ,guten ' die Kampfstätte geschlagen verließen, hat Gewalt an sich in Kriminal- und Westernfilmen oft Erfolg. Das bedeutende Ergebnis einer BBC-Studie besteht darin, daß der Aggressor in Kämpfen mit einem klaren Ergebnis große Aussicht hatte, von der Gewalt zu profitieren. "11 In pastoraler Sicht kann, ungeachtet dieser Feststellung, darauf verwiesen werden, daß es Erlebnisse gibt, die sich wie Gift in der Seele festfressen. Da das Friedrich Nietzsche (1844-1900) zugeschriebene Wort „Tugend ist Mangel an Gelegenheit" zufolge der Erfahrung einen gewissen Wahrheitsgehalt hat, müßte man bei der Beurteilung der Inhibitions-These zwischen Langzeit- und Kurzzeitwirkung unterscheiden. Die Kirche könnte pastoral auf Wirkungen der Phantasie hinweisen, etwa nach dem Jesuslogion: „Wer eine Frau auch 
nur lüstern ansieht, hat in Gedanken schon Ehebruch mit ihr begangen" (Mt. 5,28). Die heute oft vergessene Wahrheit, daß man sich in der Phantasie ebenso nicht alles erlauben darf wie beim Essen und Trinken, könnte so wieder in Erinnerung gerufen werden.

\section{Habitualisierungs-These}

Nach dieser These hat Mediengewalt eine Gewöhnung zur Folge, die zu kontinuierlich abnehmenden und schließlich ausbleibenden aggressiven Emotionen und Verhaltenstendenzen führt. Von der Lernpsychologie her ist jedoch eine andere Deutung wahrscheinlicher: Wo bereits genügend aggressives Verhalten und entsprechende Tendenzen erlernt worden sind, ist allmählich kein zusätzliches Lernen mehr möglich. Hierauf hinzuweisen, könnte auch eine kirchliche Aufgabe sein.

\section{Stimulations-These}

Mediengewalt wird hier gedeutet als einladend zur Nachahmung. Wenn auch nur ein kleiner Personenkreis dafür in Frage kommen sollte, so dürfte dieser bei der hohen Fernsehdichte groß genug sein, um mögliche Verbrechen aufkommen zu lassen. Alarmierend ist die Beobachtung von Nicholas Johnson, nach der nach Einführung amerikanischer Western- und Kriminalserien im Ausland regelmäßig ${ }^{12}$ fünf bis zehn Jahre später eine Welle neuer Gewalttätigkeit folgt. Nicholas Johnson ist eines der sieben Mitglieder der „Federal Communications Commission" der USA.

Da es um den Einfluß von Mediengewalt auf das jugendliche Gewissen geht, soll im folgenden der Versuch unternommen werden, die Wirklichkeit des hier gemeinten Gewissens näher zu bestimmen. „Vom Gewissen erwartet der Mensch heute seine individuelle Freiheit. Wo es sich dabei um ein recht verstandenes Unterscheiden, Sichentscheiden und Agieren in Freiheit handelt und nicht um einen Vorwand zur Willkür, ist diese Freiheit der Person nur zu erreichen im Hinsehen auf gewissenhafte Menschen und im Horchen auf den Spruch des gebildeten Gewissens. Gewissensbildung geschieht durch Vorbild und Beistand im Wertnehmen und Sichentscheiden innerhalb der mehrseitigen Relationen während des lebenslangen Reifungs- und Entfaltungsprozesses des Menschen. Die zuständigen Helfer zu Entfaltung der einzelnen Fähigkeiten in dem komplexen Phänomen Gewissen sind die Beziehungspersonen des jungen Menschen in Elternhaus, Schule und Beruf. Sie sollen ihm durch Vorbilder helfen in dem Einüben seiner persongerechten Freiheit. "13

Viele junge Leute leiden darunter, daß sie zwar zahlreiche Bekannte, aber keine echten Beziehungspersonen haben. Auch fehlt es ihnen nicht selten an entsprechenden Stellvertretern, die sie auch im Fernsehen nicht finden. Es ist das Verdienst von Wilhelm Heinen, auf die "Beziehungspersonen" hingewiesen zu haben ${ }^{14}$. Er teilt sie in "acht Grundgestalten" ein: Vater, Mutter, Bruder, Schwester, Mann, Frau, Sohn, Tochter. Nach Heinen ist der Mensch während des lebenslangen Werde- und Reifungsprozesses auf das Erleben dieser Beziehungspersonen oder deren adäquater Stellvertreter unbedingt angewiesen. Das Fehlen dieser Grundgestalten und der Stellvertreter kann 
nach Heinen mit dem Phantomschmerz verglichen werden, der in einem amputierten, nicht mehr vorhandenen Gliedmaß (Phantomglied) empfunden wird. Mit Hilfe des

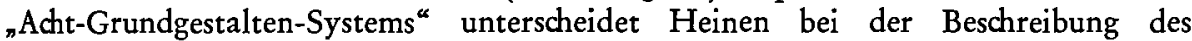
"Gewissens" zwei Komponenten: 1. Maternal: Wertsensibilität, intuierendes Werterfassen, Unterscheidungsfähigkeit von "gut" und „böse “; 2. Paternal: Entscheidungsfähigkeit, Durchhaltefähigkeit einer verantwortet getroffenene Entscheidung. Paternal und maternal meint hier: Diese Gewissenskomponenten werden überwiegend von der Mutter (maternal) bzw. vom Vater (paternal) übernommen, nachgeahmt, internalisiert. Anders ausgedrückt: Hat der Mensch in seiner frühen Kindheit Vater oder Mutter oder beide ungenügend oder kaum - und auch keine echten personalen Stellvertreter - erlebt, ist er ungenügend entfaltet in den Fähigkeiten seines Gewissens: "Zu der Entfaltung des Gewissens trägt die mater mit der Entfaltung des Wertintuierens und Wahrnehmens, der pater mit der Entfaltung des Vorbildens und Sichentscheidens bei. ${ }^{\text {"15 }}$ "Fehlen dem Kind oder Jugendlichen die Vorbilder und das Beispiel der Eltern und Geschwister, so geraten sie in einen Notstand, der vom Unbewußten mit erstaunlicher Präzision signalisiert wird (Auffälligwerden, Erkrankung, Leistungsschwund). "16 In dem Heinen-Entwurf wird die zentrale Frage des Menschen, ob und wie sein Leben gelingen kann, mit Hilfe der „acht Grundgestalten“ zu lösen versucht. „Die unbewußt gebliebenen Fragen, die unerfüllt gebliebenen Erwartungen und Forderungen des Kindes stellen sich verstärkt im Leben des Jugendlichen; sie drängen auf allen Wegen und mit allen Mitteln auf Beantwortung und Erfüllung. Jugendliche fragen direkt - und heute noch öfter indirekt - nach dem Prozeß des Werdens, menschlich und beruflich. Sie fragen nach dem Sinn und Verhalten ihrer Eltern, nach der Position und Aufgabe der Brüder und Schwestern, besonders eindringlich, wenn sie in der Ursprungsfamilie ganz oder teilweise fehlen, weil ohne diese horizontale Geschwister-Relation das Sich-Lösen von den Eltern erschwert oder behindert wird. In Frage gestellt werden die bestehenden Strukturen und Ordnungen in Familie, Staat und Kirche. Die Formen des kritischen Sondierens und In-Frage-Stellens reichen von Weigerung über Renitenz, Demonstration bis zum Aufruhr und zur Forderung nach Umsturz durch Revolution. Neben und mit diesen Formen finden sich als Ausdruck des Unbehagens, der Unzufriedenheit und der Frustration: Leistungsausfall, Erkrankungen, Unfall (Berufs-, Sport-, Verkehrsunfall), Vergehen, Delikte (Raub, Diebstahl, Gewaltverbrechen), Suizidversuch und perfekter Suizid (Selbstentbindung von Eltern, Beruf und Leben).

\section{Was fordern Jugendliche mit solchem Verhalten?}

a) $\mathrm{Daß}$ sie gesehen, gehört, angehört werden, und zwar zunächst von ihren Eltern und Geschwistern, sofern sie solche haben. Wenn diese fehlen oder unverständig sind, fordern sie Stellvertreter.

b) Jugendliche fordern, daß Eltern und Erwachsene sie verstehen in den Schwierigkeiten ihrer Entwicklung, in der Not ihres Werdens und Sichentscheidens. Mit Verstehen ist hier nicht nur ein intellektuelles Begreifen, vielmehr ein intuierendes Innewerden ihrer existentiellen Reifungsnot gemeint. Uber ein solch mittenhaft-kardiales Innewerden von Person zu Person kommt das Einvernehmen zustande, das zu dem unerläßlichen Annehmen des Du und Wir und damit des Ich-selbst führt.

c) Die Forderungen aller Jugendlichen zielen auf Vorbilder und Leitbilder, nicht nur oder primär auf Information und Wegweisung. Wenn es zutrifft, daß die für die Entfaltung des Menschen entscheidenden Lebensvollzüge und Haltungen von Mit- 
menschen abgesehen, nachvollzogen und angeeignet werden, dann ist das Bestehen auf gültigen, $d$. h. überzeugenden Vorbildern nicht nur verständlich, sondern unerläßlich. ${ }^{\text {17 }}$

Ausgehend von diesem Heinen-Modell gerät ein Jugendlicher durch den Ausfall von echten Beziehungspersonen in erhebliche Reifungsverzüge. Es scheint nahezu, als habe er eine Art „Phantom-Nerv", mit dessen Hilfe er sich seine ungenügend oder überhaupt nicht erlebten Beziehungspersonen unaufhörlich und eindringlich sucht. In dieser Situation können in der Tat Fernseh-Helden Einfluß gewinnen. Von daher kann in solchen Fällen Mediengewalt sowohl kurz- als auch langfristig eine negative Wirkung auf die Gewissensbildung des Jugendlichen haben. Für die pastorale Praxis wäre hier beispielsweise zu fordern, daß die Kirche gründlicher als bisher junge Leute auf die Ehe vorbereitet, um so „Ursprungs-Familien “ zu schaffen, die selbst innerlich geordnet sind, um „Un-Ordnung“ und somit Anfälligkeiten zu Gewissensverbildungen der Kinder zu vermeiden. Ähnliches könnte auch Bedeutung erlangen als Zusatzkriterien für die Zulassung zu den ehe-freien Diensten im Priester und Ordensstand. J. Köhne nennt einige Kriterien wie Annahme der eigenen Geschlechtlichkeit, Lösung aus kindhafter Elternbindung, Fähigkeit und Willen zur Ubernahme von Verantwortung für sich und andere, Abrücken von unbewußten Leitbildern und Wunschvorstellungen, Wissen um Eigenart und spezifische Reaktionen des anderen Geschlechtes sowie deren Akzeptierung und die Fähigkeit zum Dialog ${ }^{18}$.

Mediengewalt hat auf keinen Fall die Funktion eines versöhnenden Ersatzrituals, wenn die eigentlichen Ursachen der Störung nicht gesehen und somit nicht angegangen werden können. Entscheidend ist, daß die Wurzeln der Unzufriedenheit, Unausgeglichenheit und des Zerstrittenseins des Menschen mit Gott und mit sich selbst saniert werden können.

In Heinens ,acht Grundgestalten " wird die Möglichkeit des Gelingens des lebenslangen menschlichen Werde- und Reifungsprozesses vorausgesetzt, was selbstverständlich auch ein Scheitern mitmeinen kann. Vielleicht kann man das Geheimnis, das wir "Gott" nennen dürfen, in diesem Zusammenhang einmal "definieren" als den, der garantiert, das jedes menschliche Leben restlos (!) gelingen, aber auch scheitern kann. Zunächst kann dies bedeuten: Kein Mensch braucht seine persönlichen Hoffnungen auf eine persönliche Vollendung im Augenblick seines Todes aufzugeben. Anders ausgedrückt: Der Christ ist nicht nur auf dem Wege seiner "natürlichen", sondern darüber hinaus auch auf dem Wege zu seiner „übernatürlichen“ Vollendung. Ein Schlüsselbegriff zu dieser Argumentationsfigur scheint in den Begriffen „Bindung“ und "Lösung" zu liegen. „Bindung kann sowohl leibbetonte als auch überwiegend seelische (psychische) Abhängigkeit eines Menschen von einem anderen bezeichnen. Das allmähliche, stufenweise Eigen- oder Selbständig-Werden ist nicht ausgeschlossen, vielmehr mitgemeint und beabsichtigt..." ... "Lösung und Ablösung bezeichnen die seelische, emotionale Entbindung. ${ }^{\text {"10 }}$ Hier wird auf die Wandlungsnotwendigkeit und Wandlungsfähigkeit aufmerksam gemacht. Der Begriff "Wandlung“ und die damit verbundene Wirklichkeit hat während der Messe einen besonderen Sinn, wobei

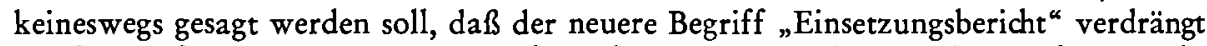
werden muß. In unserem Zusammenhang kann genügen: Zum Gelingen des menschlichen Lebens während des lebenslangen Werde- und Reifungsprozesses gehört die „Wandlungsfähigkeit ${ }^{\alpha}$; erfahrungsgemäß geht das nicht ohne Schmerz ab. Vielleicht könnte der Sinn des Meßopfers unter diesem Gesichtspunkt mitbedacht werden: Um "eucharistie-fähig" zu werden, muß der Christ bereit sein, sich von Christus „wandeln“ 
zu lassen; er darf sich weder an Personen noch an anderes unnötig festklammern. Nach dem Heinen-Modell könnte das heißen, daß sich jeder Mensch altersgemäß von bestimmten "Grundgestalten" trennen muß, um für die nächsten dazusein. Das Possessivpronomen „mein “ kann dies verdeutlichen helfen: "mein“ ist nicht "mein"; wenn eine Mutter zu ihrem Säugling „mein“ sagt, so ist das etwas anderes, als wenn sie das zu ihrem erwachsenen Sohn sagt, wobei dennoch ein „Kern“ bleibt: Ob Säugling oder erwachsener Sohn - es ist und bleibt der Mutter unbenommen, „mein“ zu sagen, wenn auch mit unterschiedlichen Akzenten. Wenn die „Lösung“, die „Wandlung“, jedesmal erreicht wurde, so erlebt der Mensch eine größere Welt und somit eine Art Belohnung für seine "Lösung" und „Wandlung“. Wenn das irdische Leben zu Ende geht, im Tod, kann das eine zusätzliche Begründung zu der Hoffnung sein, die Christus durch sein Leben und Sterben erworben hat: die Hoffnung auf Unsterblichkeit. Jede Art von „Lösung“, "Wandlung“ und "Uberwindung“ könnte so als eine Art Training gesehen werden, den Tod nicht als Ex-Itus, sondern als Trans-Itus zu sehen und $\mathrm{zu}$ bejahen. Taufe, Firmung und Eucharistie als Initiationssakramente der katholischen Kirche könnten in diesem Sinne ihren Höhepunkt im christlichen Todesverständnis erreichen: Hier im Tod geschieht $\mathrm{kraft}$ des Christus-Ereignisses die „Lösung “, die „Wandlung“ und somit der Trans-Itus. Von der christlichen Verkündigung hier ist es gewiß nicht schwer, Gott als jene Wirklichkeit zu sehen, die die "Garantie ${ }^{\star}$ dafür gibt, daß jedes menschliche Leben restlos gelingen kann. Es geht also nicht nur um die natürliche, sondern um die Vorbereitung auf die übernatürliche Vollendung in Gott. Diese Gedanken können eine Zusatzhilfe sein, den Tod christlich zu verstehen: Der Glaube an die Auferstehung muß u. a. auch gesehen werden als Protest gegen die Vorstellung, daß menschliches Leben für immer erlischt. Das sei auch in Richtung jener Ideologien gesagt, die mehr oder weniger bewußt den Menschen im Tod als eine Art "Revolutions-Müll“ enden sehen, insofern er zwar eine bessere Zukunft mitvorbereitet, diese jedoch nie erreichen wird, weil man nicht an Gott glauben kann oder will. Gegenüber diesen Ideologien könnte die Kirche zusätzlich geltend machen, daß der, der alles „verändern“ will, sich nicht selten scheut vor der eigenen (!) Wandlung: Das Reich Gottes ist nahe, kehrt um (Mk 1,15)!

Wie wirkt Mediengewalt auf die Gewissensbildung der Jugendlichen?

Die Kirche sollte hier ihre Chancen nutzen, dem jungen Menschen zu dem zu verhelfen, was er wirklich zur Erreichung seiner natürlichen und übernatürlichen Vollendung benötigt. Einiges wurde dazu gesagt. Immerhin: Auch heute erwarten viele von der Kirche Antwort und Hilfen, auch zu diesen Fragen. Möge die Kirche ihren Kairos erkennen!

\section{Anmerkungen:}

1. Erklärung vom 28. Oktober 1965 über die christliche Erziehung „Gravissimum Educationis", Nr. 1.

2. Nach Brodkhaus Enzyklopädie, Wiesbaden 1968 ff., Bd. IV, S. 167: im Jahre $1960=$ 4.635.000 Fernsehgeräte $=1,6$ Geräte je 8,6 Einwohner, $1968=14.141 .282$ Geräte; vermuteter Sättigungsgrad bei 80-85\%. 1977 $=19.987 .061$ (angemeldete) TV-Geräte: nach „Media Perspektiven“, Nr. 3/77, S. 176. 
3. Nach den ZDF-Jahrbüchern 1971 bzw. 1976 (S. 188-191 bzw. 165-167) sieht das Zuschauerinteresse auszugsweise so aus:

Nacbrichten/Politik

heute (montags bis freitags)

1971

1976

ZDF-Magazin

$17 \%$

$24 \%$

$19 \%$

$14 \%$

Bilanz

$13 \%$

$13 \%$

Ortszeit

$8 \%$

Kennzeichen D 10\%

Sport

Aktuelles Sportstudio

$17 \%$

$19 \%$

Sport-Spiegel

$7 \%$

$19 \%$

Unterbaltung

Der Kommissar (Kriminalfilm) $\quad 74 \%(!)$

$66 \%$

Wünsch Dir was

$39 \%$

Der große Preis $57 \%$

4. Quelle: Fernsehen in den 70er Jahren, Schriftenreihe des ZDF, Heft 9, Mainz 1971, S. 22, und „Media Perspektiven“, Nr. 5/75, S. 193.

5. Rede anläßlich der Verleihung des Friedenspreises des Börsenvereins des Deutschen Buchhandels 1969 in Frankfurt; veröffentlicht in "Der Spiegel“, Nr. 42, 1969, S. 206-212.

6. Hemmerle/Hagemann: Kirchen - wollt ihr euch behaupten? München-Zürich-Wien 1970, S. 26.

6². Martin Buber: Die fünf Bücher der Weisung ... Köln ${ }^{3} 1968$, S. 209.

7. Aufbau der Person, München ${ }^{9} 1964$, S. 288.

8. A. J. Yates, in: WilhelmArnold u. a. (Hrsg.): Lexikon der Psychologie, Bd. I/2, Freiburg 1976, Sp. 657.

9. Nr. 10.

10. Berkowitz, in: Wilhelm Arnold u. a. (Hrsg.): Lexikon der Psychologie, Bd. I/1, Freiburg 1976, Sp. 36.

11. Heiko Flottau: Moderne Märchen auf der Mattscheibe? In: „Süddeutsche Zeitung“, Nr. 131 vom 10./11. Juni 1972, S. 3.

12. Ebd.

13. W. Heinen: Das Gewissen - sein Werden und Wirken zur Freiheit bearbeitet und herausgegeben von Hans Kramer, Würzburg 21971, S. 7.

14. W. Heinen: Begründung christlicher Moral. Beiträge zur Moralanthropologie, Würzburg 1969, S. 40.

15. A.a.O., S. 32.

16. Ebd., S. 60.

17. W. Heinen, in: J. Tenzler (Hrsg.): Urbild und Abglanz, Beiträge zu einer Synopse von Weltgestalt und Glaubenswirklichkeit, Regensburg 1972, S. 240 f.

18. J. Köhne: Entwicklung menschlicher Geschlechtlichkeit als Voraussetzung für die Ehe, in: Albrecht Beckel (Hrsg.): Ehe im Umbruch, Münster 1969, S. 47-52.

19. W. Heinen: Begründung christlicher Moral, Würzburg 1969, S. $120 \mathrm{f}$.

\section{S U M M A R Y}

According to the documents of the Second Vatican Council, especially "Gravissimum Educationis" and "Inter Mirifica", the Church is responsible for the formation of the conscience of young people in regard to violence on TV. After treating three theories on the origin of 
aggression (innate; from frustration; an acquired habit) the author deals with the possible effects of violence in the media under the aspects of catharsis, inhibitions, habit-forming or stimulation theses. According to the ideas of Wilhelm Heinen, Professor of Moral Theology, the succes of a lifelong growth-process depends on the experience of eight basic personal relationships in human life: to father, mother, brother, sister, husband, wife, son and daughter. When relationships with these persons or their substitutes are not created, then maturity is retarded, either overtly or unconsciously. If a young person has not sufficient experience of basic family relationships, then exposure to violence on TV will probably produce a harmful effect. According to the proposals made by Heinen, it is the pastoral duty of the Church to build up or substitute for basic human relationships.

\section{RESUME}

Selon les dires du Concile Vatican II (Gravissimum Educationis, Inter Mirifica), l'Eglise est responsable de la formation de conscience de la jeunesse face aux représentations de la violence à la télévision. Après une représentation des trois théories sur l'origine de l'aggression (tendance innée, conséquence de la frustration, attitude apprise), il est question des effets des représentations de la violence dans les mass media dans la thèse de la catharsis, de l'inhibition, de l'habituation et de la stimulation. D'après le modèle de Wilhelm Heinen, la réussite du processus de devenir et de maturité, tout au long de la vie, dépend de l'expérience des huit formes de base des personnes de relation père, mère, frère, soeur, homme, femme, fils, fille. $\mathrm{Si}$ aucunes relations ne sont créées ici, alors naissent des résidus de maturité qui se signalent de façon consciente, et plus encore inconsciente. En cas de famille d'origine insuffisamment vécue, la représentation de la violence par les mass media a des effets négatifs sur la jeunesse. Il est du devoir pastoral de l'Eglise de créer des relations correspondantes dans le sens des „huit statures de base“ de Heinen et de ses représentants.

\section{RESUMEN}

Según el Concilio Vaticano II (Gravissimum Educationis, Inter Mirifica) la Iglesia es responsable de la formación de la conciencia de la juventud ante la violencia propagada por la televisión. Tras la presentación de las tres teorías sobre el origen de la agresividad (impulso innato, frustración, actitud adquirida) se trata de las repercusiones en la tesis de la catarsis, la inhibición, la habituación y del estímulo de la presencia de la violencia en los medios de difusión. Según modelo de Wilhelm Heinen el proceso siempre incompleto de la madurez depende de la experiencia vital en relación con ocho personas: padre, madre, hermano, hermana, marido, esposa, hijo, hija. Si no existen relaciones vitales de este tipo se producen lagunas de madurez, que actúan en el consciente y más aún en el subconsciente. Cuando se dan insuficiencias experimentales de este tipo, la violencia presentada por los medios de difusión tiene repercusiones negativas en la juventud. La Iglesia tiene la misión pastoral de crear las relaciones correspondientes en el sentido de los „ocho elementos“ de Heinen ó sus sucedáneos. 